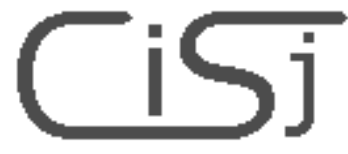

computing@tanet.edu.te.ua www.tanet.edu.te.ua/computing
ISSN 1727-6209

International Scientific

Journal of Computing

\title{
ОБЪЕКТНОЕ ПРЕДСТАВЛЕНИЕ КОНТУРА УПРАВЛЕНИЯ ДИСКРЕТНЫМИ ПРОЦЕССАМИ НА ОСНОВЕ СИСТЕМ ПРОДУКЦИЙ
}

\section{Тихомирова Е.В.}

\author{
Белорусский государственный университет \\ информатики и радиоэлектроники, \\ г. Минск
}

Резюме: Рассматривается задача программной реализации контура управления дискретными процессами в системах организационно-технологического уровня с применением технологии объектно-ориентированного моделирования. В качестве базового формализма при решении задачи используются системь продукиий. Вопросы интерпретации системы продукции решаются по схеме интерпретации процессов на сетевой структуре.

Ключевые слова: логическое управление, дискретные процессы, сети Петри, системы продукиий.

\section{ВВЕДЕНИЕ}

Возросший повсеместно интерес к проблемам построения высокоэффективных и высоконадежных систем автоматизированного управления, например в промышленности, транспорте, энергетике и т.д., способствовал широкому применению методов моделирования для исследования функционирования и поведения сложных объектов [1]. В общем виде процесс взаимодействия управляющей части с объектом управления и внешней средой может быть охарактеризован системой функциональных зависимостей $F$ :

$$
Y=F(X) \text {, }
$$

где $X=\left\{x_{1}, x_{2}, \ldots x_{n}\right\}$ - входные данные, представляющие собой контролируемые состояния объекта управления или внешней среды;

$Y=\left\{y_{1}, y_{2}, \ldots, y_{m}\right\}-$ выходные данные, например, управляющие воздействия на объект управления.

Функционирование системы заключается в регулярном фиксировании фактов изменения переменных состояний системы с последующим рекуррентным изменением значений функционально - зависящих от них объектов представления выходных данных. Известны способы формализации таких систем, например автоматные модели, графы операций [2, 3, 4], однако способ их дальнейшего представления в процедурном виде не обсуждался.

Задача разработки способа представления в памяти ЭВМ зависимостей, отражающих процесс функционирования системы и проведения анализа этих зависимости в ускоренном и реальном режиме времени здесь будет рассматриваться с позиции технологии объектно-ориентированного моделирования.

\section{1. ДИСКРЕТИЗАЦИЯ ЗАКОНОВ УПРАВЛЕНИЯ В ТЕРМИНАХ СЕТЕВЫХ МОДЕЛЕЙ}

Пусть система характеризуется тройкой:

$$
S=\langle V, P, A\rangle,
$$

где $V-$ множество переменных состояния системы $\left(V_{i} \in V\right)$;

$P$ - множество условий на $V$, задаваемых вычислимыми предикатами;

$A$ - множество действий, соответствующих условиям $P$.

Каждое действие $A-$ множество преобразований вида $V:=A(V)$, причем правые части этих преобразований обязательно вычислимы на $V$, а все члены правой части подвергаются преобразованию одновременно.

Любой элемент $V_{i}$ из множества переменных состояний системы $V$, может быть охарактеризован четверкой функциональных зависимостей

$$
V_{i}=\left(E_{i}, C_{i}, F_{i}, D_{i}\right),
$$

где $E_{l}$ - условия активизации элемента $E_{i} \in P$;

$C_{i}$ - действия, вызываемые в системе при входе элемента в активное состояние, $C_{i} \in A$;

$F_{i}$ - действие перехода при выходе элемента из активного состояния $F_{i} \in A$; 
$D_{i}$ - длительность во времени активной фазы элемента.

Процесс функционирования системы представляет собой регулярное применение процедур реализации элементарных действий из множества $C$ (рис.1).

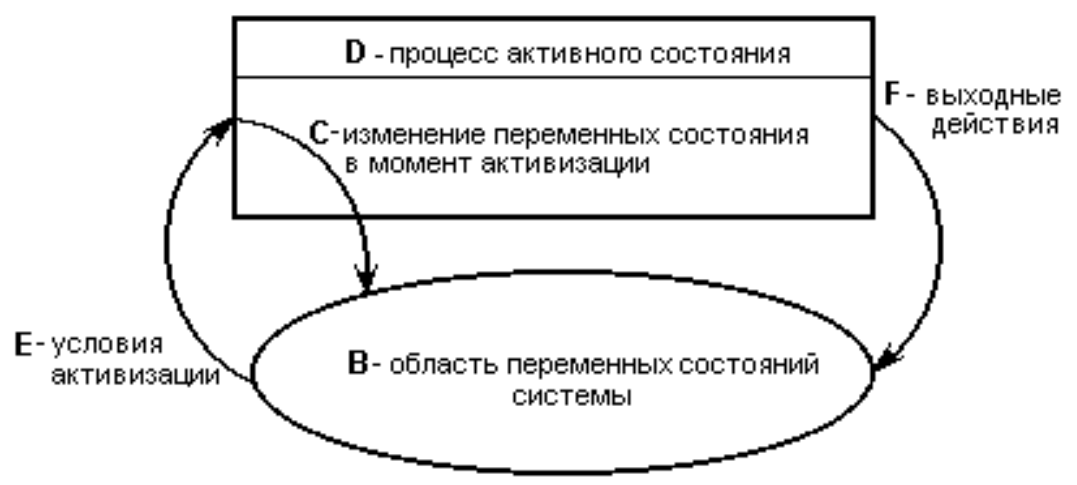

Рис.1. Графическое представление процесса функционирования системы.

Подходящим методом математического описания системы для последующего представления в ЭВМ является система продукций, представляющая собой набор продукционных правил:

$$
\text { ЕСЛИ <условие }>\text { ТО }\langle\text { действие }>\text {. }
$$

Система продукционных правил описывает множество объектов моделируемой системы и отношения между ними. Такое представление моделируемой системы удобно тем, что позволяет:

- однозначно фиксировать объекты и их свойства, действия над ними;

- специалисту (конструктору, технологу) сформулировать правила решений (поведения системы) в привычном для него представлении, т. е. в форме, близкой к справочникам, стандартам и другим источникам, что позволяет в дальнейшем избежать сложности формализации поведения объектов системы;

- модульность продукций дает возможность расширения и корректировки данной среды с учетом конкретной специфики.

Однако, формальное представление функционирования системы в виде продукционных правил не отражает динамики связи переменных состояний системы во времени. Нетрудно заметить, что задача интерпретации системы продукции - получение последовательности действий может решаться по схеме интерпретации процессов на временной сети Петри $(T P N)$ в виде последовательности активизации переходов [5, 6]. Например, рассмотрим сеть Петри (рис.2)

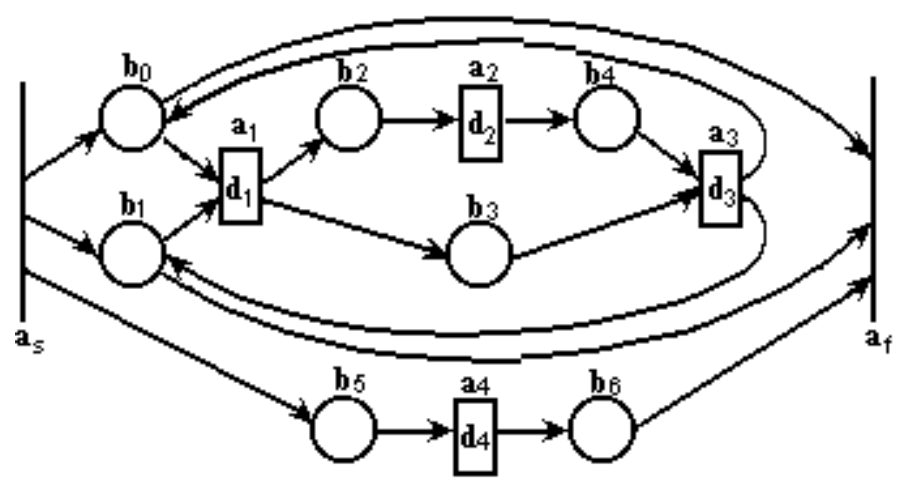

Рис.2. Графическое представление сети Петри

Введем обозначения:

$b_{1}, b_{2}, \ldots, b_{n}$ - переменные состояния $b_{i} \in B$,

$a_{1}, a_{2}, \ldots, a_{m}$ - действия $a_{i} \in A$,
$E, C$ и $F-$ функции связывающие разметку позиций с весами дуг сети.

Сеть TPN может быть представлена структурой смежности: 
выходные связи позиций:

$$
\begin{aligned}
& b_{0}: a_{f}, a_{1} \\
& b_{1}: a_{f} ; a_{1} \\
& b_{2}: a_{2} \\
& b_{3}: a_{3} \\
& b_{4}: a_{3} \\
& b_{5}: a_{4} \\
& b_{6}: a_{f}
\end{aligned}
$$

выходные связи переходов:

$$
\begin{aligned}
& a_{s}: b_{0}, b_{1}, b_{5} \\
& a_{1}: b_{2}, b_{3} \\
& a_{2}: b_{4} \\
& a_{3}: b_{0}, b_{1} \\
& a_{4}: b_{6}
\end{aligned}
$$

В случае TPN позиции соответствуют переменным состояния, а переходы - действиям системы. Если некоторый переход $a_{i}$ стал пассивным, то это порождает необходимость проверки активизации переходов $\left\{a_{i+1}\right\}$ множества функций $E_{i+1}$ которые содержат переменные измененные функцией $F_{i}$. Если условие $E_{i+1}$ истинно, то выполняются действия $C_{i+1}$ после чего активизируется $a_{i+1}$ и проверяется возможность активизации переходов, условия активизации которых определены и на переменных функции $C_{i+1}$.

Таким образом, порождение процесса активизации переходов основано на восприимчивости отдельных переходов системы продукций к изменению только локальных переменных состояния. Отсюда следует, что на переходах системы продукций можно формально построить сеть (рис.3), связывающие переходы с потенциальной возможностью активизации.

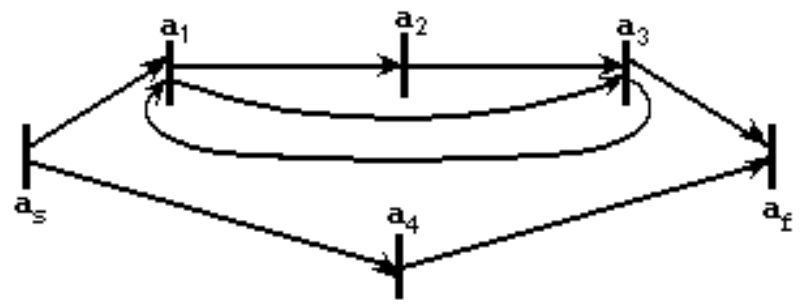

Рис. 3. Пример сетевого преобразования системы продукций.

Структура смежности связей переходов сети системы продукций (рис.3):

$$
\begin{aligned}
& a_{s}: a_{1}, a_{4} \\
& a_{1}: a_{2}, a_{3} \\
& a_{2}: a_{3} \\
& a_{3}: a_{f}, a_{1} \\
& a_{4}: a_{f}
\end{aligned}
$$

Определения функций для сетевого преобразования системы продукций :

$$
\begin{aligned}
& E\left(a_{s}\right)=0 ; \\
& E\left(a_{1}\right)=b_{0} \& \& b_{1} ; \\
& E\left(a_{2}\right)=b_{2} ; \\
& E\left(a_{3}\right)=b_{3} \& \& b_{4} ; \\
& E\left(a_{3}\right)=b_{5} ; \\
& E\left(a_{f}\right)=b_{0} \& \& b_{1} \& \& b_{6} ;
\end{aligned}
$$

$$
\begin{aligned}
& C\left(a_{s}\right)=0 ; \\
& C\left(a_{1}\right)=b_{0}--, b_{1}--; \\
& C\left(a_{2}\right)=b_{2}--; \\
& C\left(a_{3}\right)=b_{3}--, b_{4}--; \\
& C\left(a_{3}\right)=b_{5}--; \\
& C\left(a_{f}\right)=b_{0}^{--}, b_{1}--, b_{6}--;
\end{aligned}
$$

$D\left(a_{s}\right)=0$

$D\left(a_{1}\right)=d_{1}$;

$D\left(a_{2}\right)=d_{2}$

$D\left(a_{3}\right)=d_{3}$;

$D\left(a_{3}\right)=d_{4}$

$D\left(a_{f}\right)=0$;

Таким образом, система продукции может быть представлена массивом функции:

$$
\left\{a_{i}:<E_{i}, C_{i}, F_{i}, D_{i}>, i=1, \ldots, m\right\}
$$

$$
\begin{aligned}
& F\left(a_{s}\right)=b_{0}++, b_{1}++, b_{5}++; \\
& F\left(a_{1}\right)=b_{2}++, b_{3}++; \\
& F\left(a_{2}\right)=b_{4}++; \\
& F\left(a_{3}\right)=b_{0}++, b_{1}++; \\
& F\left(a_{3}\right)=b_{6}++; \\
& F\left(a_{f}\right)=0 ;
\end{aligned}
$$

и так называемой интерпретируемой сетью переходов. Такую структуру можно интерпретировать как переходную систему. 


\section{2. АЛГОРИТМИЧЕСКАЯ ИНТЕРПРЕТАЦИЯ СЕТЕВЫХ МОДЕЛЕЙ СРЕДСТВАМИ ЯЗЫКА С++}

Из удобства содержательной интерпретации

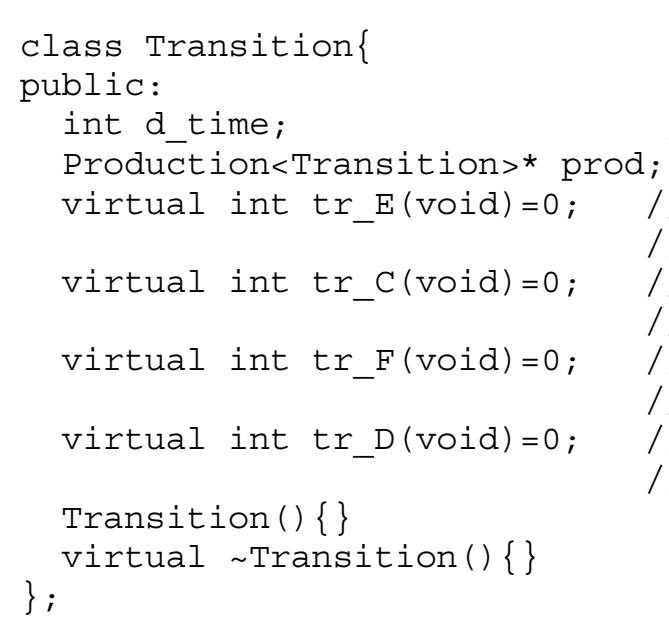

Условия активизации перехода могут определяться константой, логическим выражением или обращением к функции. Длительность задержки переходов в активизированном состоянии допускается задавать константой, переменной либо выражением. Функции и действия переходов могут включать любые операторы языка $\mathrm{C}++$ и в частности $E$ и $C$ могут включать операторы деклараций объектов. Интервал активности перехода ограничен моментами вызова процедур элементарному действию на сети идентифицированный одношаговый процесс во времени - переход, характеризуемый четверкой функциональных зависимостей общего вида $a_{i}:<E_{i}, C_{i}, F_{i}, D_{i}>$ :
$E$ и $C$ и по определению перехода оперативно привязан к временной оси. Однако не существует препятствий для изменения плана завершения активной фазы перехода. Подобные действия может выполнять другой переход, который скорректирует список событий на интерпретируемой сети.

Статическое описание сети представлено параметризованной структурой Frame, содержащей определение схемы связей переходов и позиций:

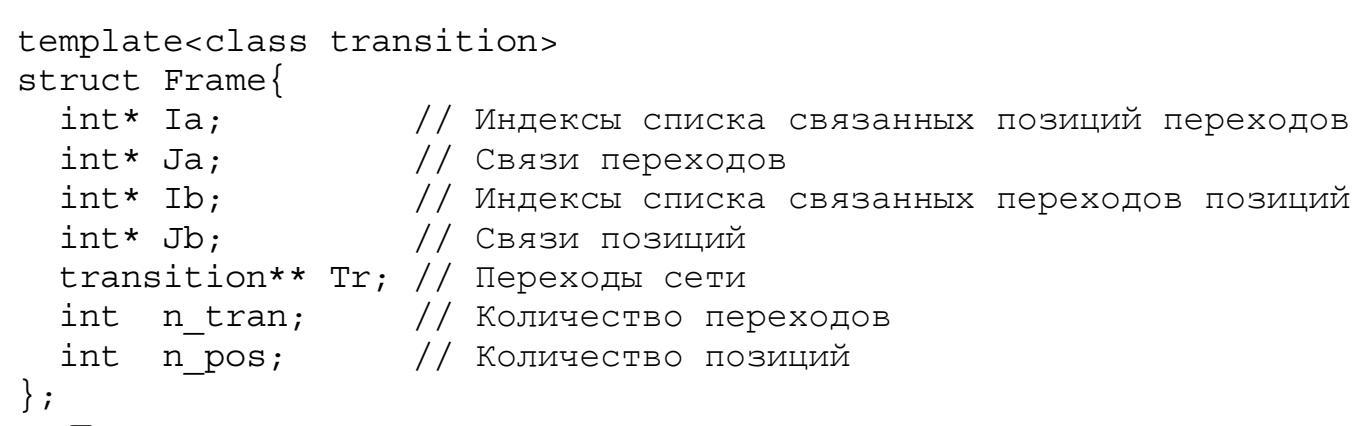

Динамическое описание сети осуществляется в классе TPN :

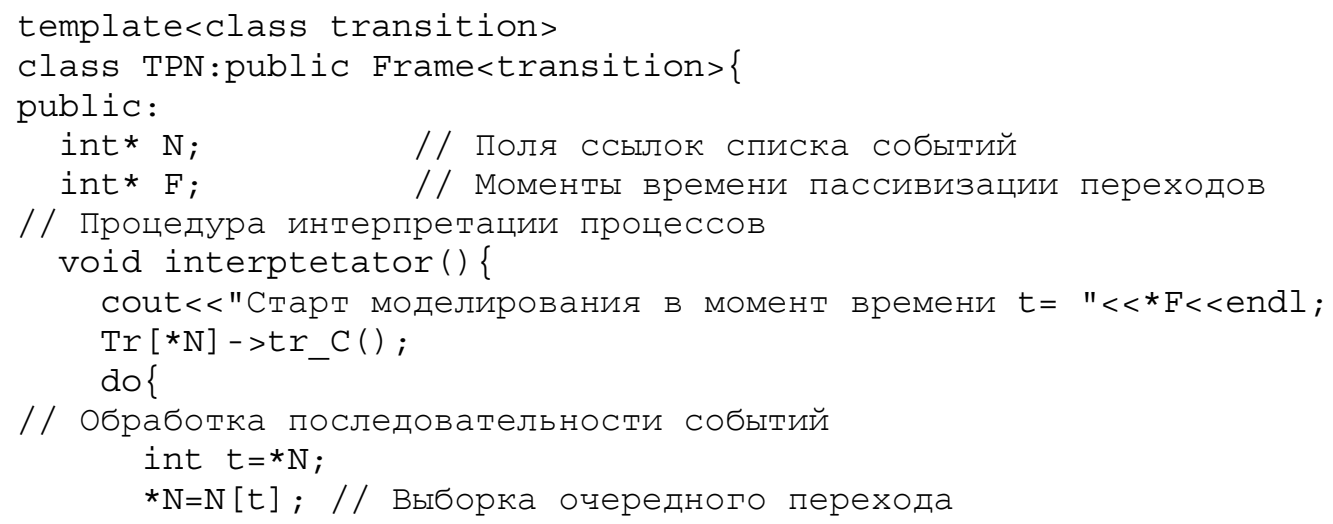
связей 


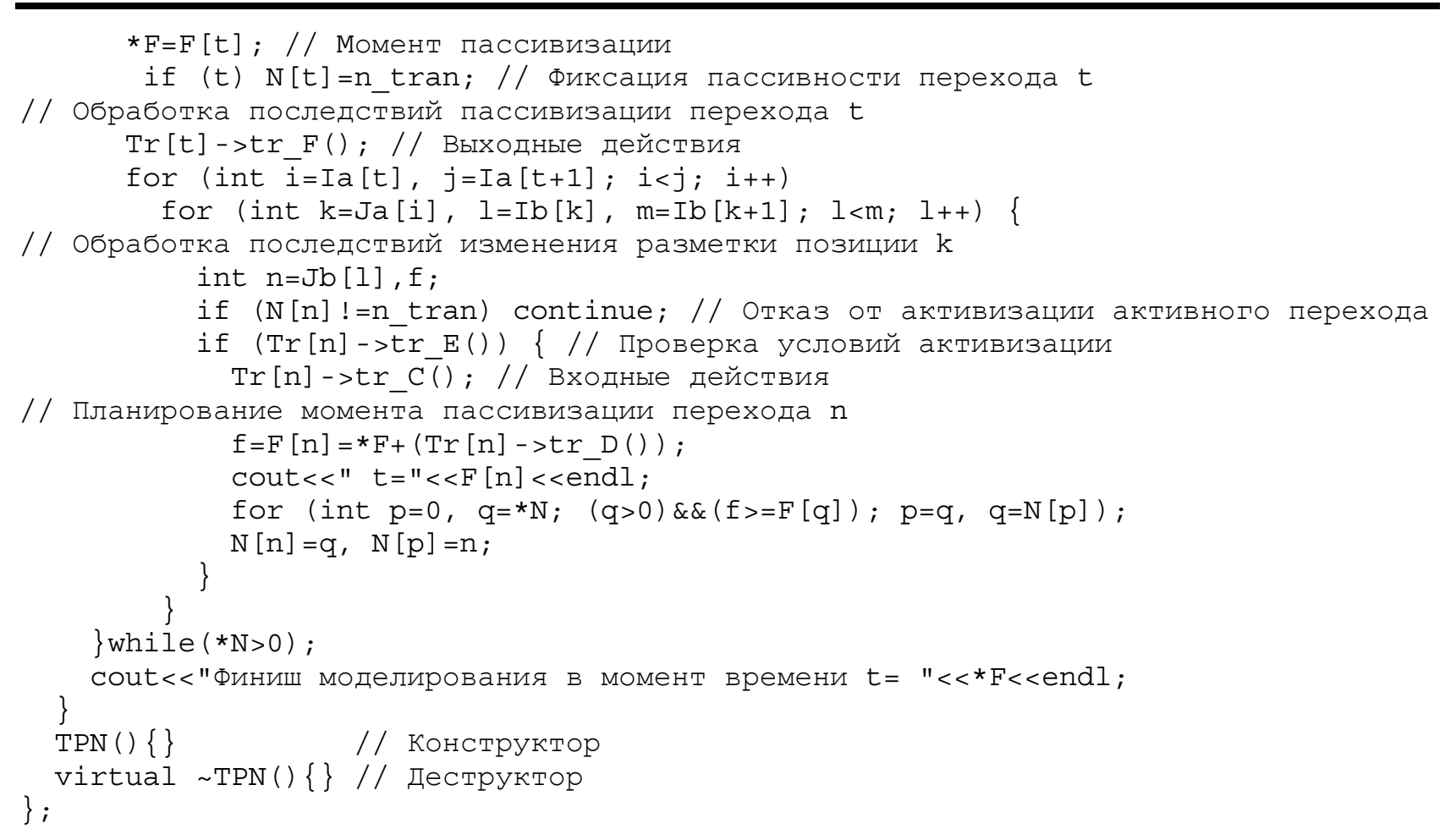

\section{Класс интерпретации системы продукций :}

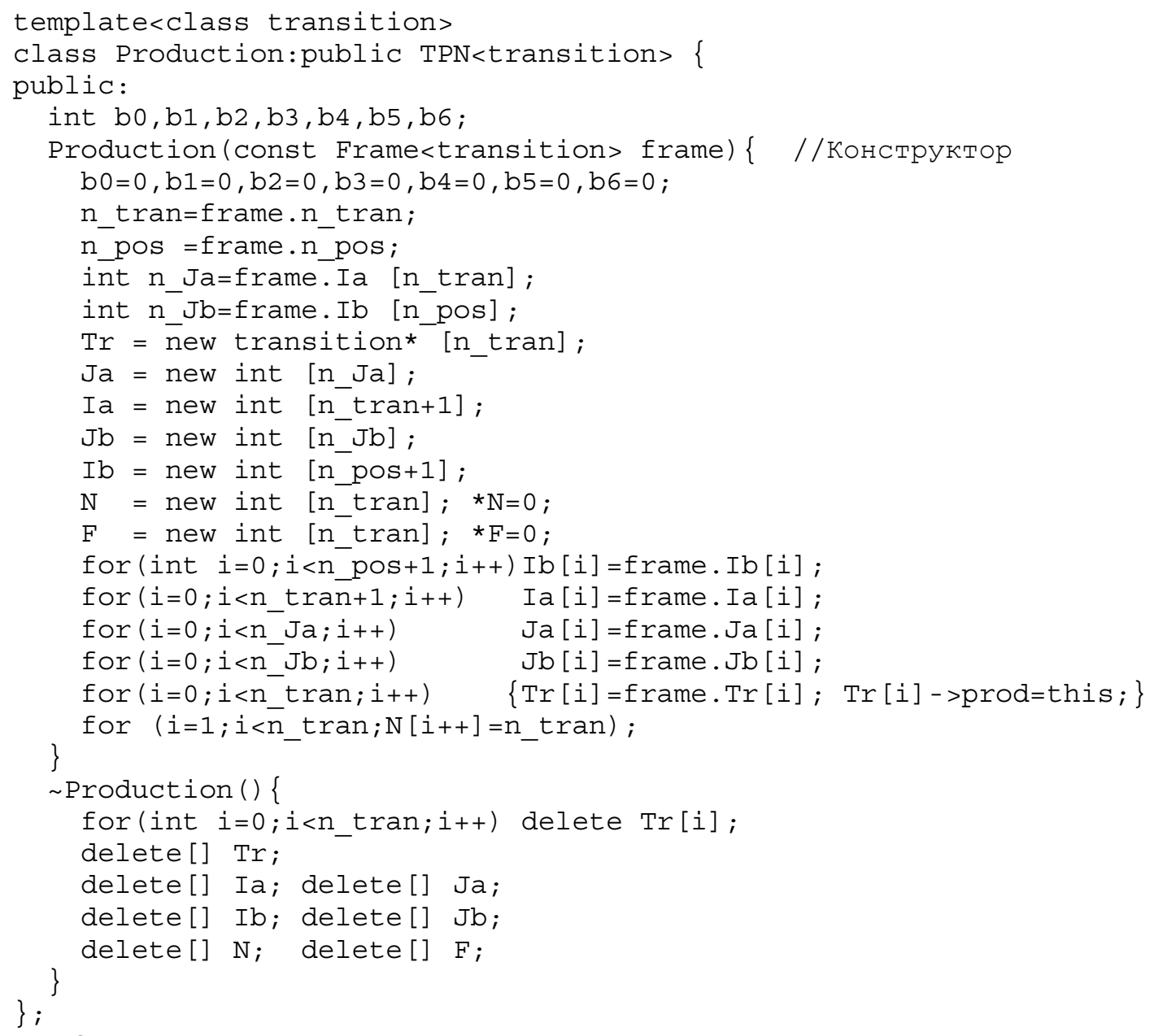

Определим классы переходов сети системы продукций (рис.3):

class Transition_as:public Transition \{ public:

virtual int tr_E(void) $\{\operatorname{return} 0 ;\}$ 


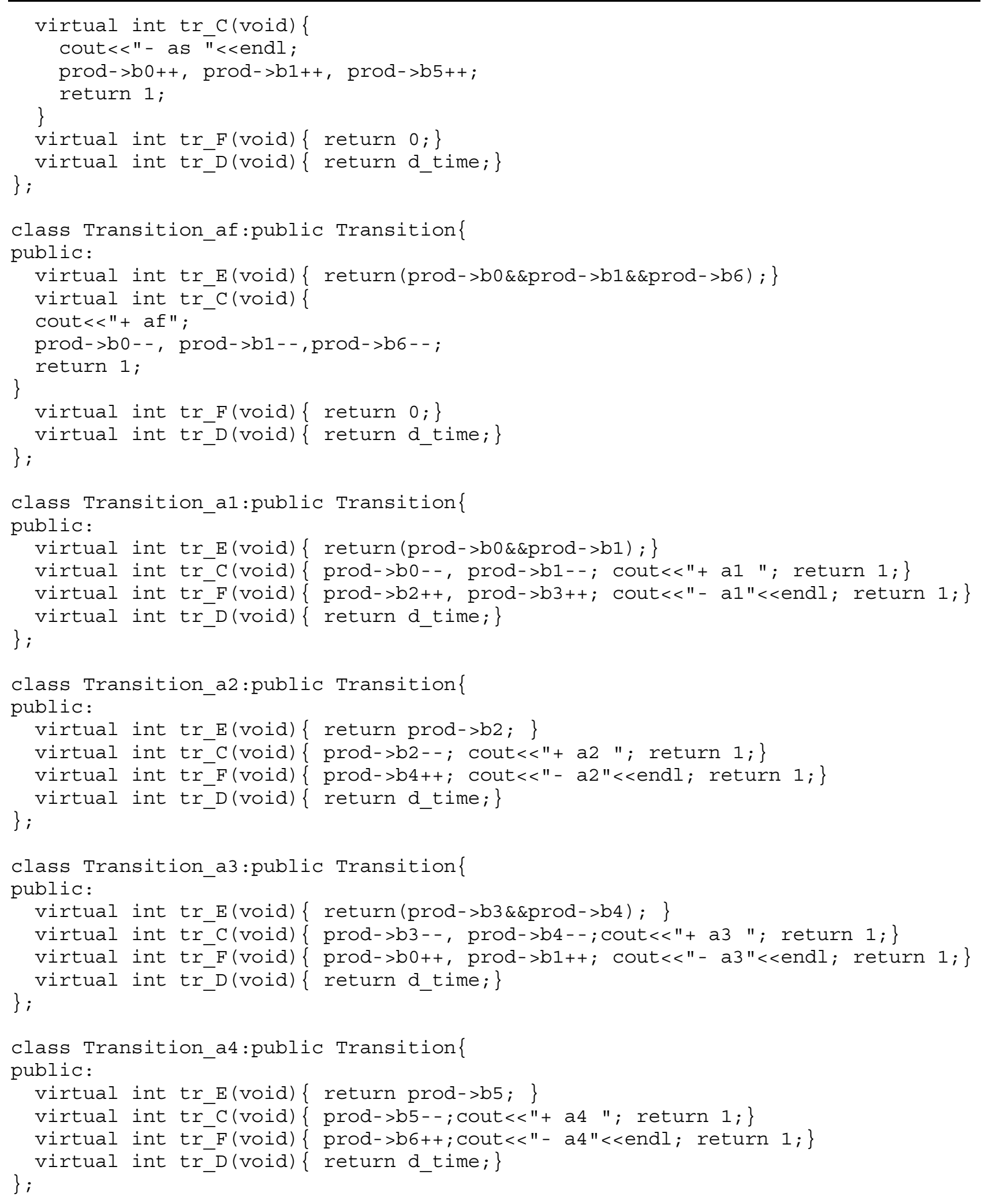

Фрагмент программы использования разработанных классов:

void main (void) \{

//...

// Схема связей переходов

// Индексы списка связанных позиций переходов

int $\operatorname{Iax}[]=\{0,7,10,14,16,20,22\}$;

//Связи переходов

int $\operatorname{Jax}[]=\{0,1,2,3,4,5,6,0,1,6,0,1,2,3,2,4,3,4,0,1,5,6\}$; 


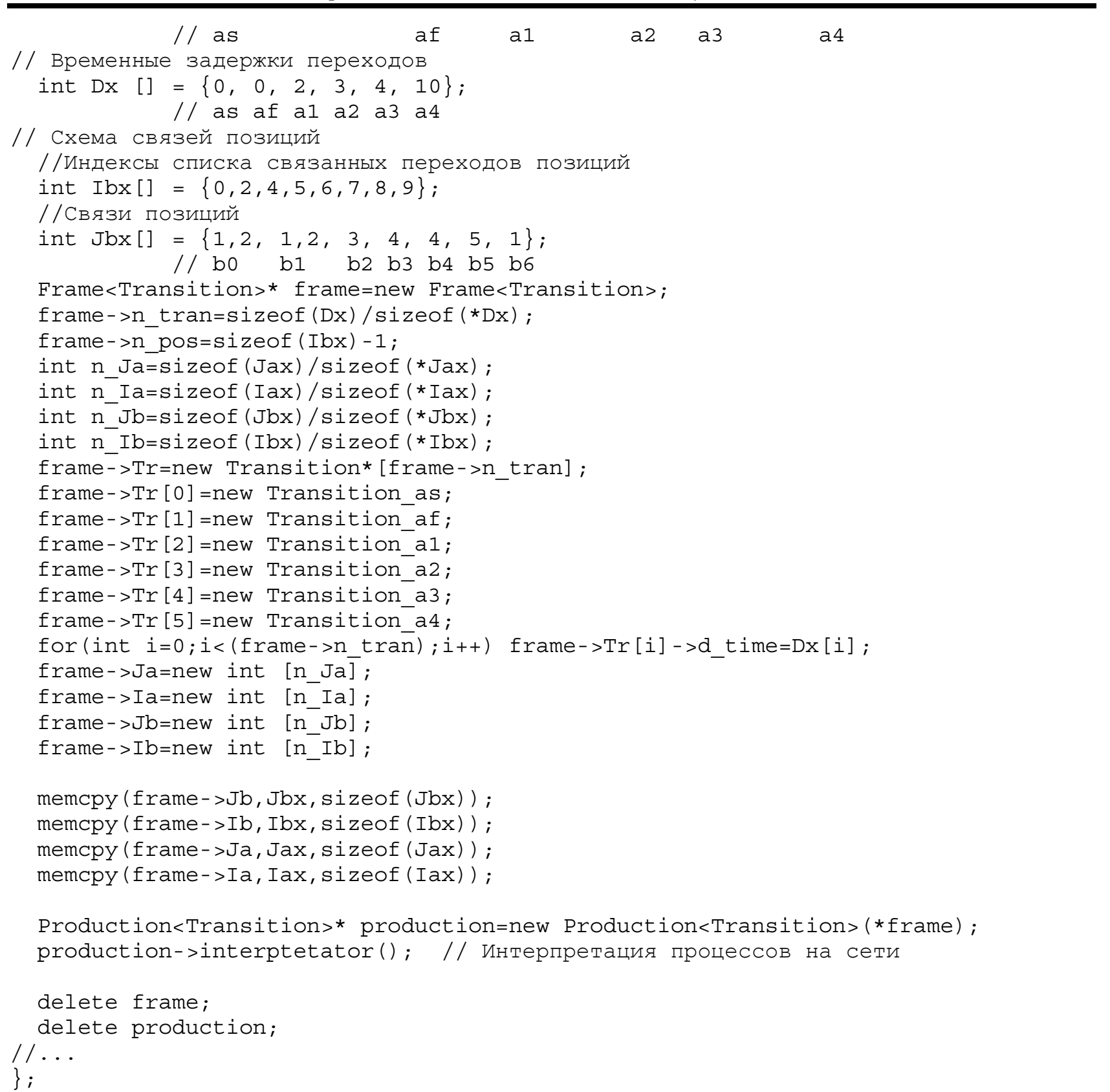

Результаты работы имитационной программы:

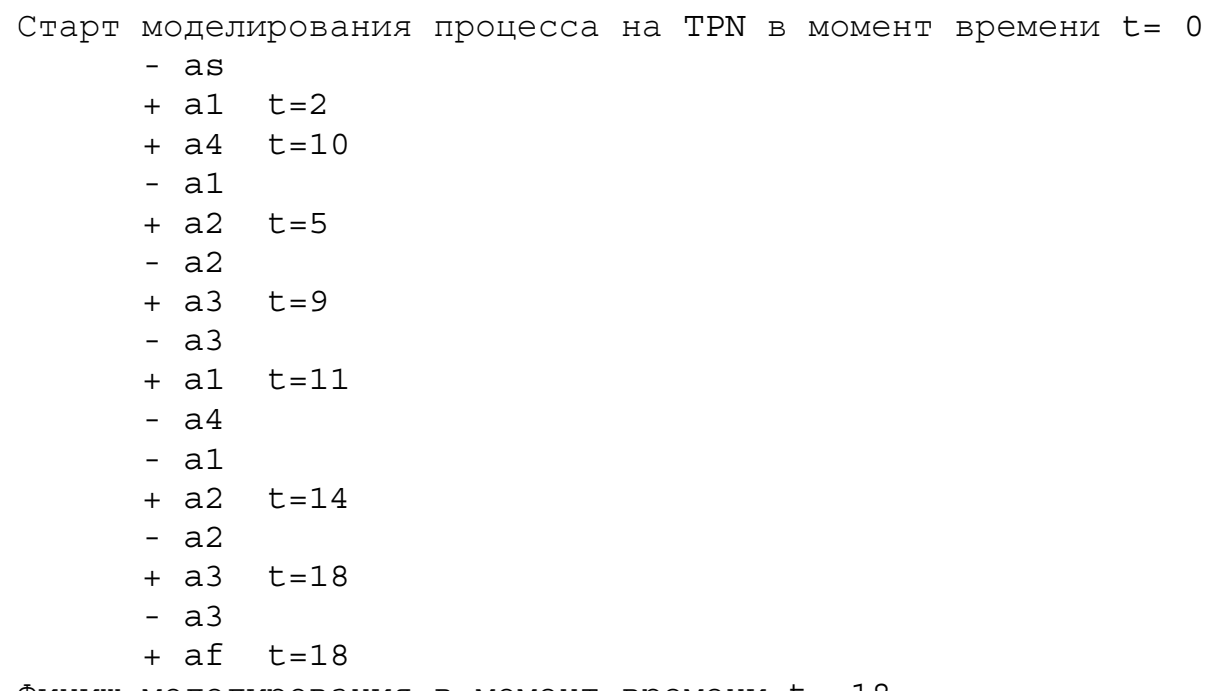

Финиш моделирования в момент времени $t=18$ 
Таким образом, полученные результаты полностью соответствуют результатам интерпретации представленной рассматриваемой системой продукций $T P N$.

\section{ЗАКЛЮЧЕНИЕ}

Рассмотренный способ представления систем с дискретным характером поведения позволяет автоматизировать переход от их формального описания к программной реализации контура управления.

Предложенный алгоритм моделирования процессов на сетевых моделях допускает оценку эффективности ядра контура управления по критерию “память-быстродействие", что может быть использовано для конструктивного решения задач компенсации погрешностей воспроизведения законов управления в реальном времени.

\section{ЛИТЕРАТУРА}

1. Бусленко Н.П. Моделирование сложных систем. - М.: Наука, 1978. - 400 c.

2. Мальшев Н.Г. Структурно-автоматные модели технических систем. М.:Радио и связь, 1986. - 168c.

3. Юдиџкий С.А., Магергут В.3. Логическое управление дискретными прочессами. - М.: Машиностроение, 1987. - 175 c.

4. Питерсон Дж. Теория сетей Петри и моделирование систем. М.: Мир, 1984. $264 c$.
5. Ревотюк М.П. Моделирование и оптимизация управления дискретными прочессами на основе интерпретируемых сетей. Диссертация на соискание ученой степени кандидата технических наук. - Мн.: МРТИ, 1989, - 120c.

6. Тихомирова Е.В. Объектная интерпретаџия систем продукиий средствами языка C++ //Радиомир. Ваш компьютер, 2000.- №11.- C. 32-35.

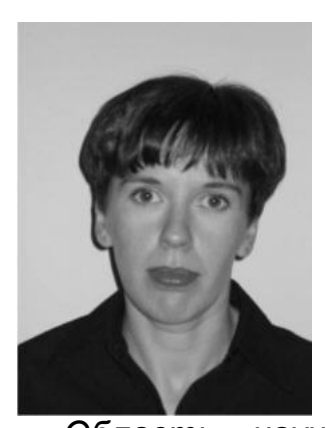

Екатерина Тихомирова работает системным программистом "WaySystems Inc.", Medford USA.

Кандидат технических наук с 20022 по специальности 05.13.01 - Cистемный анализ, управление и обработка инфрормации (промышленность).

Область научных интересов: объектноориентированное моделирование и проектирование систем управления, методы спецификация контура управления на основе переходных систем, алгоритмы интерпретации дискретных процессов на сетевых структурах, криптографичческие схемы и алгоритмы, Smart card технологии, архитектуры и модели иифровых сотовых телекоммуникационных систем, методы и алгоритмы разработки протоколов связи для беспроводных мобильных устройств. 